\title{
Production of Ginsenoside-Rg $\mathrm{g}_{3}$ from Lipomyces starkeyi Grown on Ginseng-Steam- ing Effluent
}

\author{
Jeong-Hoon Jang', Na-Mi Kim ${ }^{2}$ and Jong-Soo Lee ${ }^{1 *}$ \\ ${ }^{\prime}$ Department of Life Science and Genetic Engineering, Paichai University, Daejeon 302-735, Korea \\ ${ }^{2}$ Korean Ginseng Corporation, Central Research Institute, Daejeon 305-345, Korea
}

(Received March 30, 2010. Accepted April 19, 2010)

To produce ginsenoside-Rg $g_{3}$ enriched yeast from ginseng-steaming effluent (GSE), Lipomyces starkeyi, which tends to grow

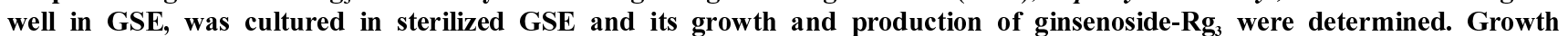
of $L$. starkeyi was $86.1 \mathrm{mg}$ per $\mathrm{g}$ GSE and its ginsenoside- $\operatorname{Rg}_{3}$ contents was $0.013 \mathrm{mg}$ per $\mathrm{g}$ GSE.

$\overline{\text { KEYWORDS : Ginseng-steaming effluent, Ginsenoside-Rg }}$, Lipomyces starkeyi

Recently, scientists have begun to use various yeasts for the production of bioactive compounds, such as an antihypertensive angiotensin I-converting enzyme inhibitor $[1,2]$ and an antiangiogenic compound [3]. Ginsenoside- $\operatorname{Rg}_{3}$ [4] and antidementia $\beta$-secretase inhibitor [5] have also been produced and characterized from Saccharomyces cerevisiae.

Ginseng and its extracts are used in the production of various health foods and neutriceuticals. From the manufacturing process of ginseng products there is a large amount of ginseng steaming effluent (GSE) that is discharged as waste [6]. GSE, however, contains useful ginsenosides, sugars, protein and industrial enzymes and has potential physiological functionality [7]. Only a small amount of GSE is necessary for the extraction of useful ginsenosides or in the production of bioactive malto-oligosaccharides [6], chitosan [8] and ribonucleotides [9]. The bulk of GSE is discharged into the sewage, causing environmental pollution. We studied the production of bioactive compounds from GSE and herein report on the production and characterization of ribonucleotides from Pichia anomala [9] and its mutant [10], chitosan by Mucor miehei [8] and ginsenoside- $\operatorname{Rg}_{3}$ [4] from $S$. cerevisiae grown on GSE. We now describe production of ginsenoside- $\mathrm{Rg}_{3}$ enriched Lipomyces starkeyi, using ginseng-steaming effluent.

The L. starkeyi used in this study was obtained from the Bioresource Center of the Korean Research Institute of Bioscience and Biotechnology (KRIBB, Daejeon, Korea). The GSE was obtained from a ginseng processing plant in Geumsan, of Chungnam province, South Korea. This GSE contained $63.8 \%$ total sugar, $34.2 \%$ crude protein, $0.2 \%$ crude fat and $1.8 \%$ ash, and it had a $\mathrm{pH}$ of 6.5 . Ginsenoside standard products and ginsenoside analysis reagents were purchased from Sigma Chemical Co. (St. Louis,

\footnotetext{
$\overline{\text { *Corresponding author }}<$ E-mail : biotech8@pcu.ac.kr>
}

MO, USA). Unless otherwise specified, all chemicals were of analytical grade. P. anomala KCCM 11473 and L. starkeyi, grown on yeast extract-peptone-dextrose (YEPD) medium at $30^{\circ} \mathrm{C}$ for 2 days were inoculated in the GSE (pH 6.5) and cultured at $30^{\circ} \mathrm{C}$ for $72 \mathrm{hr}$. After centrifugation of the culture broth at $10,000 \mathrm{~g}$ for $15 \mathrm{~min}$, the yeast cells were harvested, sonicated and centrifuged again in order to obtain the cell-free extracts.

Determination of growth and ginsenoside contents was carried out according to the method of Kim et al. [4], with slight modifications. Growth of $L$. starkeyi in the GSE was $86.1 \mathrm{mg}$ per $\mathrm{g}$ GSE $\left(0.52, \mathrm{~A}_{660}\right)$. Growth of $L$. starkeyi was lower than that of $S$. cerevisiae grown on $\operatorname{GSE}\left(1.38, \mathrm{~A}_{600}\right)$ [4].

We determined the ginsenoside content of cell-free extract from L. starkeyi grown on GSE. As shown in Fig. 1, ginsenoside- $\mathrm{Rg}_{3}$ was identified in HPLC chromatogram of $L$. starkeyi grown on GSE and the other ginsenosides were not detected. The result was same as those of $S$. cerevisiae, Saccharomyces pastorianus, Kluyveromyces fragilis and Zygosaccharomyces rouxii [4]. Ginsenoside contents in L. starkeyi was analyzed and compared with those of GSE itself and with the yeasts grown on a YEPD medium (Table 1). Compared to the $9.66 \mathrm{mg}$ of ginsenoside- $\mathrm{Rg}_{3} / \mathrm{g}$ GSE, which was contained in GSE itself, L. starkeyi grown on GSE $(1 \mathrm{~g})$ contained $0.013 \mathrm{mg}$ of ginsenoside- $\mathrm{Rg}_{3} / \mathrm{g}$ GSE. Any ginsenosides were not dectected in cell-free extracts from the yeast grown on YEPD medium (data not shown). The ginsenoside- $\operatorname{Rg}_{3}$ content of $L$. starkeyi was lower than that of $S$. cerevisiae, K. flagilis and Zygosacch. rouxii [4]. L. starkeyi is known for intracellular fat production. There is little information on the production of some bioactive compounds from L. starkeyi; this is the first report that ginsenoside- $\operatorname{Rg}_{3}$ was produced from the yeast.

It is known that $\operatorname{Rg}_{3}$ have many valuable attributes, such as anti-cancer, anti-dementia and anti-stress proper- 

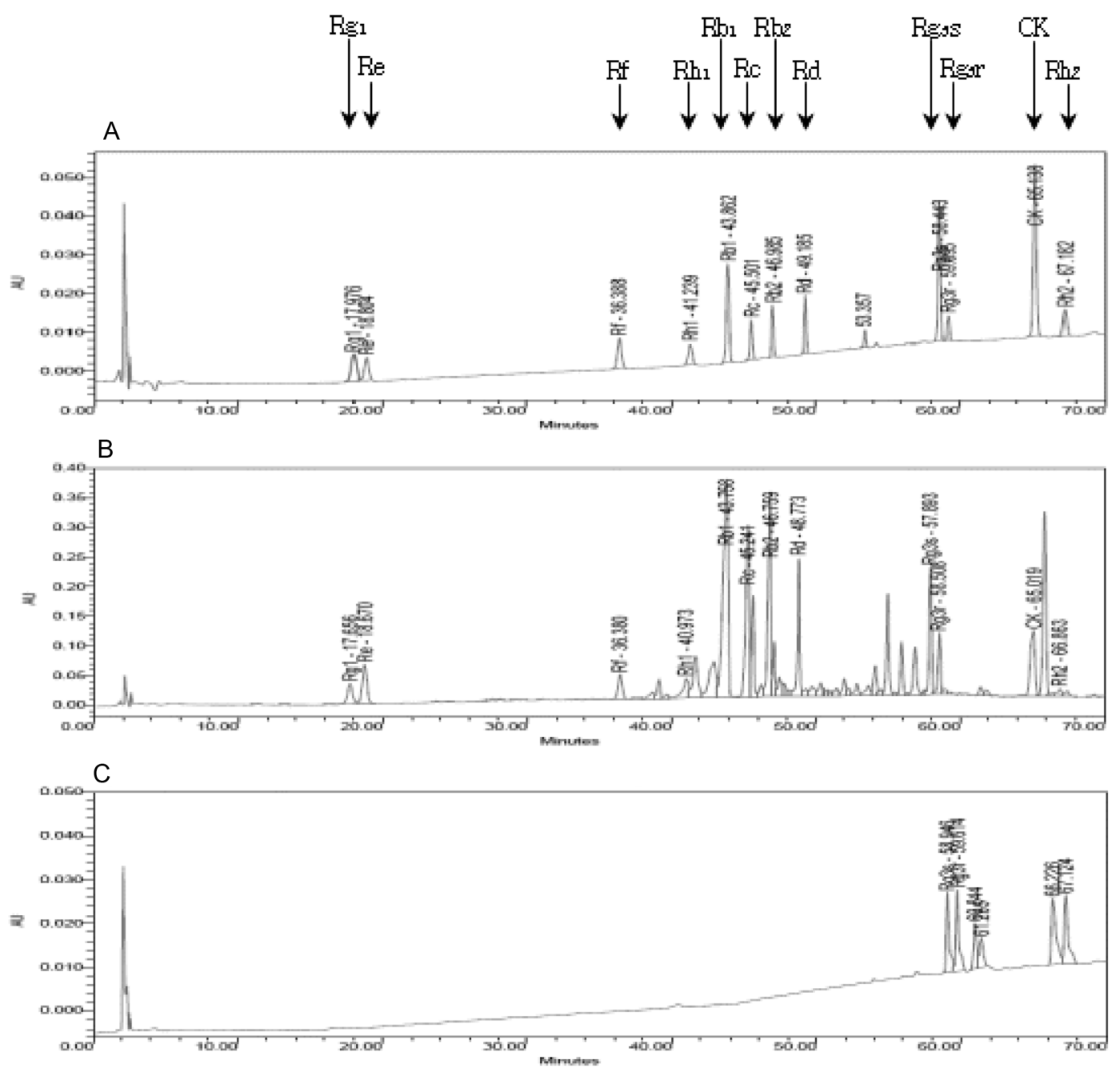

Fig. 1. HPLC chromatogram of ginsenosides. A, Ginsenoside standard; B, Ginseng - steaming effluent (GSE); C, Lipomyces starkeyi grown on GSE.

Table 1. Ginsenoside contents of Lipomyces starkeyi grown on the ginseng-steaming effluent (mg/g solid)

\begin{tabular}{ccccccccccc}
\hline & \multicolumn{10}{c}{ Ginsenosides } \\
\cline { 2 - 13 } & $\mathrm{Rb}_{1}$ & $\mathrm{Rb}_{2}$ & $\mathrm{Rc}$ & $\mathrm{Rd}$ & $\mathrm{Re}$ & $\mathrm{Rf}$ & $\mathrm{Rg}_{1}$ & $\mathrm{Rg}_{3}$ & $\mathrm{Rh}_{2}$ & $\mathrm{Total}$ \\
\hline GSE $^{\mathrm{a}}$ & 29.91 & 15.19 & 16.78 & 7.99 & 4.68 & 2.28 & 2.25 & 9.66 & 1.92 & 90.66 \\
L. starkeyi & - & - & - & - & - & - & - & 0.013 & - & 0.013 \\
\hline
\end{tabular}

GSE, ginseng-steaming effluent.

${ }^{\mathrm{a}} \mathrm{Rg}_{3}$ contents of ginseng - steaming effluent itself.

ties [4]. Also, it is converted from $\mathrm{Rb}_{1}, \mathrm{Rb}_{2}$ and $\mathrm{Rc}$ by treatment of slight acid or heat $\left(100^{\circ} \mathrm{C}, 20 \mathrm{~min}\right)$ [4]. It is presumed that ginsenoside- $\mathrm{Rg}_{3}$ of $L$. starkeyi grown on GSE in this study was produced from sterilization $\left(121^{\circ} \mathrm{C}\right.$, $20 \mathrm{~min}$ ) of GSE or bioconversion of the yeast during cultivation. Further study is need to illustrate the $\mathrm{Rg}_{3}$ production mechanism in these yeast.

\section{References}

1. Kim JH, Lee DH, Jeong SC, Chung KS, Lee JS. Character- ization of antihypertensive angiotensin 1-converting enzyme inhibitor from Saccharomyces cerevisiae. J Microbiol Biotechnol 2004;14:1318-23.

2. Lee JS, Yi SH, Kwon SJ, Ahn C, Yoo JY. Enzyme activities and physiological functionality of yeasts from traditional Meju. Kor J Appl Microbiol Biotechnol 1997;25:448-53.

3. Jeong SC, Lee DH, Lee JS. Production and characterization of an anti-angiogenic agent from Saccharomyces cerevisiae K-7. J Microbiol Biotechnol 2006;16:1904-11.

4. Kim NM, Lee SK, Cho HH, So SH, Jang DP, Han ST, et al. Production of ginsenoside- $\mathrm{Rg}_{3}$ enriched yeast biomass using ginseng steaming effluent. J Ginseng Res 2009;33:183-8. 
5. Lee DH, Lee DH, Lee JS. Production and characterization of antidementia â-secretase inhibitor from Saccharomyces cerevisiae. In: 2006 Aunnual Meeting and International Symposium; 2006 Jun 18-20; Busan, Korea. Seoul: The Korean Society for Microbiology and Biotechnology.

6. Kim NM, Lee JS, Lee BH. Enzymatic hydrolysis of Korean ginseng starch and characteristics of produced maltooligosaccharides. J Ginseng Res 2000;24:41-5.

7. Kim NM, So SH, Lee SG, Song JE, Seo DS, Lee JS. Physiological functionality and enzyme activity of biomass from Pichia anomala grown on ginseng-steaming effluent. Myco- biology 2008;36:148-51.

8. Kim JH, Lee KS, Kim NM, Lee JS. Production and characterization of chitosan from ginseng-steaming effluents by Mucor miehei. J Microbiol Biotechnol 2002;12:760-5.

9. Kim JH, Lee BH, Lee JS. Production of ribonucleotides by autolysis of Hansenula anomala grown on Korean ginseng steaming effluent. J Biosci Bioeng 2002;93:318-21.

10. Lee JS, Hyun KW, Jeung SC, Kim JH, Choi YJ, Miguez CB. Production of ribonucleotides by autolysis of Pichia anomala mutant and some physiological activities. Can J Microbiol 2004;50:489-92. 